\title{
Identification of Unstable Systems using Output Error and Box-Jenkins Model Structures
}

\author{
Urban Forssell and Lennart Ljung \\ Department of Electrical Engineering \\ Linkping University, S-581 83 Linkping, Sweden \\ WWW: http://www. control.isy.liu.se \\ Email: ufo@isy.liu.se, ljung@isy.liu.se
}

1997-12-01

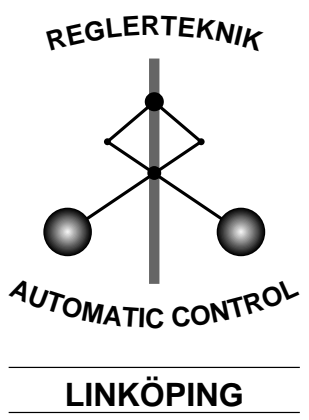

Report no.: LiTH-ISY-R-1988

Submitted to IEEE Transactions on Automatic Control.

Technical reports from the Automatic Control group in Linkping are available by anonymous ftp at the address 130.236.20.24 (ftp.control.isy.liu.se). This report is contained in the compressed postscript file $1988 \cdot \mathrm{ps} . \mathrm{Z}$. 


\title{
Identification of Unstable Systems using Output Error and Box-Jenkins Model Structures
}

\author{
Urban Forssell and Lennart Ljung*
}

December 4, 1997

\begin{abstract}
It is well known that for prediction error identification of unstable systems the output error and BoxJenkins model structures cannot be used. The reason for this is that the predictors in this case generically will be unstable. Typically this is handled by projecting the parameter vector into the region of stability which gives erroneous results when the underlying system is unstable. The main contribution of this work is that we derive modified, but asymptotically equivalent, versions of these model structures that can be applied also in the case of unstable systems.
\end{abstract}

\section{Introduction}

In this note we will discuss prediction error identification of unstable systems using output error and BoxJenkins model structures. As is well known from text books on system identification in the prediction error framework (e.g., $[4,7])$, it is required that the predictors are stable. In case the parameters are estimated using some numerical search algorithm that requires gradients of the predictor to be computed, these must also be stable. Suppose now that we want to use an output error, or a Box-Jenkins, model structure and that the underlying system is unstable. Then the predictors will generically be unstable which seemingly makes these model structures useless in these

${ }^{*}$ U. Forssell and L. Ljung are both with the Division of Automatic Control, Department of Electrical Engineering, Linköping University, S-581 83 Linköping, Sweden. Email: ufo@isy.liu.se, ljung@isy.liu.se. cases. However, as we shall see it is possible to reparameterize these model structures to cope also with unstable systems, and this without increasing the total number of parameters to be estimated.

An implicit assumption is here that the experimental data are generated with a stabilizing controller in the loop. We are thus faced with a closed-loop identification problem. Closed-loop identification is often used in connection to so called control-relevant identification where the goal is to estimate models that are suitable for (robust) control design, see, e.g., the surveys $[2,8,9]$. It is then often only interesting to model the dynamics of the plant, the noise properties are less interesting, so that it would be natural to use an output error model structure. However, since unstable plants cannot be handled using output error models the conclusion has been that this approach cannot be used when the plant is unstable. Alternative solutions have been suggested in, e.g., $[3,10]$. Unfortunately these methods are considerably more involved than a direct application of an output error or a Box-Jenkins model to the closed-loop data.

A problem when identifying systems in closedloop directly is that the results will be biased unless the noise model accurately describes the true noise characteristics [4-6]. This has traditionally been a main issue in the closed-loop identification literature that has further motivated the search for alternative closed-loop identification methods. The bias problems will of course also be present when using the new model structures suggested in this paper although in many cases the bias error will be negligible even if the noise model is incorrect. One reason for this is that in practice the model errors will be due to both bias and 
variance errors [4] and if a reasonably flexible noise model is used the bias error due to the feedback will typically be small compared to the variance error. The variance error also increases with the number of parameters which favors model structures with few parameters. We would also like to point out that most other closed-loop identification methods, that are designed to give unbiased estimates, give higher variance errors than the direct method [1]. This is an issue that in our opinion has received too little attention in the closed-loop identification literature.

The rest of the paper is organized as follows. First, in Section 2, we study some basic facts on prediction error methods and in Section 3 discuss some standard choices of model structures. This section also contains an illustration of the problems faced with when trying to identify unstable systems using output error models. Section 4 contains the main result of the paper: How the standard output error model structure should be modified to cope also with unstable systems. After introducing some additional notation we present the basic idea and go through the derivation of the required gradient filters in some detail. This is then followed by a simulation study that illustrates the feasibility of the idea. Before concluding we, in Section 5, briefly mention the corresponding changes of the Box-Jenkins model structure that are necessary to make it applicable to unstable systems.

\section{Some Basics in Prediction Error Identification}

In prediction error identification one typically considers linear model structures parameterized in terms of a parameter vector $\theta$ :

$$
y(t)=G(q, \theta) u(t)+H(q, \theta) e(t)
$$

Here $G(q, \theta)$ and $H(q, \theta)$ are rational functions of $q^{-1}$, the unit delay operator $\left(q^{-1} u(t)=u(t-1)\right.$, etc. $)$ parameterized in terms of $\theta ; y(t)$ is the output; $u(t)$ is the input; $e(t)$ is white noise.

Typically $\theta$ ranges over some open subset $D_{\mathcal{M}}$ of $\mathbb{R}^{d}(d=\operatorname{dim} \theta)$ :

$$
\theta \in D_{\mathcal{M}} \subset \mathbb{R}^{d}
$$

Note the distinction between a model and a model structure: When viewed as a function of $\theta,(1)$ is a model structure, while for a fixed $\theta=\theta^{*},(1)$ is a model. Furthermore, we also have that (1) together with (2) defines a model set and it is our task to find the best model in the set, typically by performing a numerical search over all possible models. Refer to [4] for a comprehensive treatment, including exact definitions, of the concepts model, model structure and model set.

The one-step-ahead predictor for (1) is

$$
\hat{y}(t \mid \theta)=H^{-1}(q, \theta) G(q, \theta) u(t)+\left(1-H^{-1}(q, \theta)\right) y(t)
$$

Here it is required that the filters $H^{-1}(q, \theta) G(q, \theta)$ and $\left(1-H^{-1}(q, \theta)\right)$ are stable for the predictor to be well defined. It is easy to see that this calls for an inversely stable noise model $H(q, \theta)$ and that the unstable poles of $G(q, \theta)$ are also poles of $H(q, \theta)$. These issues will play important roles in this paper.

The prediction errors $\varepsilon(t, \theta)=y(t)-\hat{y}(t \mid \theta)$ corresponding to the predictor (3) are

$$
\varepsilon(t, \theta)=H^{-1}(q, \theta)(y(t)-G(q, \theta) u(t))
$$

We will also use the following notation for the gradient of $\hat{y}(t \mid \theta)$ :

$$
\psi(t, \theta)=\frac{d}{d \theta} \hat{y}(t \mid \theta)\left(=-\frac{d}{d \theta} \varepsilon(t, \theta)\right)
$$

In the standard case of lest-squares prediction error identification one calculates the parameter estimate as the minimizing argument of the criterion function

$$
V_{N}(\theta)=\frac{1}{N} \sum_{t=1}^{N} \frac{1}{2} \varepsilon^{2}(t, \theta)
$$

Typically one finds the estimate through some numerical search routine of the form

$$
\hat{\theta}_{N}^{(i+1)}=\hat{\theta}_{N}^{(i)}-\mu_{N}^{(i)}\left[R_{N}^{(i)}\right]^{-1} V_{N}^{\prime}\left(\theta_{N}^{(i)}\right)
$$

where $V_{N}^{\prime}$ denotes the gradient of the criterion function, $R_{N}$ is a matrix that modifies the search direction and $\mu_{N}$ a scaling factor that determines the step 
length. From (5) we see that

$$
V_{N}^{\prime}(\theta)=-\frac{1}{N} \sum_{t=1}^{N} \psi(t, \theta) \varepsilon(t, \theta)
$$

and typically $R_{N}$ is chosen approximately equal to the Hessian $V_{N}^{\prime \prime}$ (which would make (7) a Newton algorithm); a standard choice is

$$
R_{N}=\frac{1}{N} \sum_{t=1}^{N} \psi(t, \theta) \psi^{T}(t, \theta)+\delta I
$$

where $\delta \geq 0$ is chosen so that $R_{N}$ becomes positive definite. This is also called the Levenberg-Marquardt regularization procedure.

Clearly it is required that both the predictor (3) and the gradient (5), which have to be computed and used in the search algorithm (7), are stable. When dealing with unstable systems this introduces constraints on the possible model structures.

\section{Commonly Used Model Structures}

With these stability requirements in mind, let us now discuss some standard choices of model structures. The discussion will in the sequel be limited to the SISO case for ease of exposition.

A quite general model structure is the following [4]:

$$
A(q) y(t)=\frac{B(q)}{F(q)} u(t)+\frac{C(q)}{D(q)} e(t)
$$

where

$$
A(q)=1+a_{1} q^{-1}+\cdots+a_{n_{a}} q^{-n_{a}}
$$

and similarly for the $C, D$, and $F$ polynomials, while

$$
B(q)=q^{-n_{k}}\left(b_{0}+b_{1} q^{-1}+\cdots+b_{n_{b}} q^{-n_{b}}\right)
$$

This model structure includes some common special cases:

1. $C(q)=1, D(q)=1, F(q)=1$, an ARX model structure.
2. $D(q)=1, F(q)=1$, an ARMAX model structure.

3. $A(q)=1, C(q)=1, D(q)=1$, an output error model structure.

4. $A(q)=1$, a Box-Jenkins model structure.

A sufficient condition for the predictor and gradient filters to be stable is that $C(q) \cdot F(q)$ is stable for all $\theta \in D_{\mathcal{M}}$ (cf. Lemma 4.1 in [4]). Note that this condition is automatically satisfied for ARX models, and for ARMAX models it is sufficient that the $C$ polynomial is stable, which does not impose any stability constraints on the dynamics model. For identification of unstable system these model structures thus are natural choices.

The output error model structure has a fixed noise model $(H(q, \theta)=1)$ and is a natural choice if only a model of the system dynamics is required. In case one wants to model also the noise characteristics but do not want the noise and dynamics models to be dependent (as in the ARX and ARMAX cases) then the Box-Jenkins model structure would the one to choose. However, if the underlying system is unstable these model structures can not be used without modifications, e.g., the ones we propose in this paper. To see where the problem lies, let us study the output error case.

Suppose that we want to identify an unstable system, stabilized by some controller, and that we are only interested in modeling the dynamics with no modeling effort spent on the noise characteristics. Then the natural choice would be to use an output error model structure:

$$
y(t)=\frac{B(q)}{F(q)} u(t)+e(t)
$$

Now, since the system is unstable the predictor

$$
\hat{y}(t \mid \theta)=\frac{B(q)}{F(q)} u(t)
$$

as well as the gradient filters

$$
\begin{aligned}
\frac{\partial}{\partial b_{k}} \hat{y}(t \mid \theta) & =\frac{1}{F(q)} u(t-k) \\
\frac{\partial}{\partial f_{k}} \hat{y}(t \mid \theta) & =-\frac{B(q)}{F^{2}(q)} u(t-k)
\end{aligned}
$$


will generically be unstable. When implementing a parameter estimation algorithm for the output error case one typically secures stability in every iteration of the algorithm (7) by projecting the parameter vector into the region of stability. For unstable systems this of course leads to erroneous results.

These problems are also present when using the Box-Jenkins model structure:

$$
y(t)=\frac{B(q)}{F(q)} u(t)+\frac{C(q)}{D(q)} e(t)
$$

Also in this case one has to resort to projections into the region of stability to ensure stability of the predictors and gradient filters, which makes this model structure in its standard form useless for identification of unstable systems.

In the following sections we will describe how to modify these model structures to avoid these problems.

\section{An Alternative Output Error Model Structure}

\subsection{Some Additional Notation}

Let $F_{s}(q)\left(F_{a}(q)\right)$ be the stable (anti-stable), monic part of $F(q)$ :

$$
F(q)=F_{s}(q) F_{a}(q)
$$

and let the polynomials $F_{s}(q)$ and $F_{a}(q)$ be parameterized as

$$
\begin{aligned}
& F_{s}(q)=1+f_{s, 1} q^{-1}+\cdots+f_{s, n_{f_{s}}} q^{-n_{f_{s}}} \\
& F_{a}(q)=1+f_{a, 1} q^{-1}+\cdots+f_{a, n_{f_{a}}} q^{-n_{f_{a}}}
\end{aligned}
$$

With the notation

$$
\bar{f}_{a, k}= \begin{cases}1 & k=0 \\ f_{a, k} & 1 \leq k \leq n_{f_{a}} \\ 0 & \text { else }\end{cases}
$$

and

$$
\bar{f}_{s, k}= \begin{cases}1 & k=0 \\ f_{a, k} & 1 \leq k \leq n_{f_{s}} \\ 0 & \text { else }\end{cases}
$$

we have

$$
f_{k}=\sum_{j=0}^{n_{f}} \bar{f}_{s, j} \bar{f}_{a, k-j}, k=1,2, \ldots, n_{f}
$$

Furthermore, let $F_{a}^{*}(q)$ denote the monic, stabilized $F_{a}$-polynomial, i.e., $F_{a}^{*}(q)$ is the monic polynomial whose zeros are equal to the zeros of $F_{a}(q)$ reflected into the unit disc. In terms of $f_{a, i}$, the coefficients of $F_{a}(q)$, we can write $F_{a}^{*}(q)$ as

$$
F_{a}^{*}(q)=1+\frac{f_{a, n_{f_{a}}-1}}{f_{a, n_{f_{a}}}} q^{-1}+\cdots+\frac{1}{f_{a, n_{f_{a}}}} q^{-n_{f_{a}}}
$$

Here we have used the implicit assumption that $f_{a, n_{f_{a}}} \neq 0$.

\subsection{The Proposed Model Structure}

Now consider the following modified output error model structure:

$$
y(t)=\frac{B(q)}{F(q)} u(t)+\frac{F_{a}^{*}(q)}{F_{a}(q)} e(t)
$$

with the predictor

$$
\begin{aligned}
\hat{y}(t \mid \theta) & =\frac{F_{a}(q) B(q)}{F_{a}^{*}(q) F(q)} u(t)+\left(1-\frac{F_{a}(q)}{F_{a}^{*}(q)}\right) y(t) \\
& =\frac{B(q)}{F_{a}^{*}(q) F_{s}(q)} u(t)+\left(1-\frac{F_{a}(q)}{F_{a}^{*}(q)}\right) y(t)
\end{aligned}
$$

The difference between this model structure and the basic output error model structure (13) is thus that we have included a noise model $F_{a}^{*}(q) / F_{a}(q)$ and obtained a different "dummy" noise term

$$
\bar{e}(t)=\frac{F_{a}^{*}(q)}{F_{a}(q)} e(t)
$$

instead of just $e(t)$. At first glance it may thus seem as the model structures (13) and (24) will give different results, but in fact they are (asymptotically) equivalent as can be seen from the following result.

Proposition 1 When applying a prediction error method to the model structures (13) and (24) the resulting estimates will asymptotically, as $N \rightarrow \infty$, be the same. 
Proof 1 First we note that from classical prediction error theory we have that the limiting models will minimize the integral of the spectrum of the prediction errors. Now, if we let $\varepsilon_{F}(t, \theta)$ denote the prediction errors obtained with the model (24) and let $\varepsilon(t, \theta)$ denote the prediction errors corresponding to the model (13), we have that

$$
\begin{aligned}
\Phi_{\varepsilon_{F}}(\omega) & =\left|\frac{F_{a}\left(e^{i \omega}\right)}{F_{a}^{*}\left(e^{i \omega}\right)}\right|^{2} \Phi_{\varepsilon}(\omega) \\
& =\left|f_{a, n_{f_{a}}}\right|^{2} \Phi_{\varepsilon}(\omega)
\end{aligned}
$$

Thus the spectra differ by only a constant scaling and hence the corresponding limiting models will be the same.

As we have seen, the results will asymptotically be the same with both model structures; the difference is of course that the predictor (25) will always be stable along with all its derivatives even if $F(q)$ is unstable (as opposed to standard output error case which require a stable $F(q)$ for the predictor to be stable). Note that in (24) the noise model is monic and inversely stable and the unstable poles of the dynamics model are also poles of the noise model (cf. the discussion in Section 2).

As a last remark before turning to implementation issues we would like to point out that the basic idea behind the equivalence result above is really that a constant spectrum may be factorized in infinitely many ways using all-pass functions. Here we chose convienient pole locations for these all-pass functions to have stable predictors.

\subsection{Computation of the Gradient}

As mentioned above, the gradient $\psi(t, \theta)$ is needed for the implementation of the search scheme (7). With the predictor (25) the expression for the gradient will be much more involved than (15) but for completeness we will go through these calculations in some detail (after all, the gradient is needed for the implementation of the estimation algorithm).

Given the predictor model (25) we have that

$$
\frac{\partial}{\partial b_{k}} \hat{y}(t \mid \theta)=\frac{1}{F_{s}(q) F_{a}^{*}(q)} u(t-k)
$$

while

$$
\frac{\partial}{\partial f_{k}} \hat{y}(t \mid \theta)=\frac{\partial}{\partial f_{k}} \frac{B(q)}{F_{s}(q) F_{a}^{*}(q)} u(t)-\frac{\partial}{\partial f_{k}} \frac{F_{a}(q)}{F_{a}^{*}(q)} y(t)
$$

Introducing

$$
\begin{aligned}
W_{1}^{k}(q) & =\frac{\partial}{\partial f_{k}} F_{s}(q) \\
W_{2}^{k}(q) & =\frac{\partial}{\partial f_{k}} F_{a}(q) \\
W_{3}^{k}(q) & =\frac{\partial}{\partial f_{k}} F_{a}^{*}(q)
\end{aligned}
$$

and

$$
\begin{aligned}
& x_{1}(t)=-\frac{B(q)}{F_{s}^{2}(q) F_{a}^{*}(q)} u(t) \\
& x_{2}(t)=-\frac{1}{F_{a}^{*}(q)} y(t) \\
& x_{3}(t)=-\frac{B(q)}{F_{s}(q)\left(F_{a}^{*}(q)\right)^{2}} u(t)+\frac{F_{a}(q)}{\left(F_{a}^{*}(q)\right)^{2}} y(t)
\end{aligned}
$$

we may write

$$
\frac{\partial}{\partial f_{k}} \hat{y}(t \mid \theta)=W_{1}^{k}(q) x_{1}(t)+W_{2}^{k}(q) x_{2}(t)+W_{3}^{k}(q) x_{3}(t)
$$

What we then finally need in order to be able to compute the gradient $\frac{\partial}{\partial f_{k}} \hat{y}(t \mid \theta)$ are explicit expressions for the filters $W_{i}^{k}, i=1,2,3$. Using (22) we have that

$$
\begin{aligned}
& W_{1}^{k}(q)=\sum_{i=1}^{n_{f_{s}}} w_{1, i}^{k} q^{-i}, w_{1, i}^{k}= \begin{cases}\bar{f}_{a, k-i}^{-1} & k-n_{f_{a}} \leq i \leq k \\
0 & \text { else }\end{cases} \\
& W_{2}^{k}(q)=\sum_{i=1}^{n_{f_{a}}} w_{2, i}^{k} q^{-i}, w_{2, i}^{k}= \begin{cases}\bar{f}_{s, k-i}^{-1} & k-n_{f_{s}} \leq i \leq k \\
0 & \text { else }\end{cases}
\end{aligned}
$$


while

$$
\begin{aligned}
W_{3}^{k}(q) & =\frac{1}{f_{a, n_{f_{a}}}} \cdot\left[\sum_{i=1}^{n_{f_{a}}-1} w_{3, i}^{k} q^{-i}+w_{3,0}^{k}\left(1-F_{a}^{*}(q)\right)\right], \\
w_{3, i}^{k} & = \begin{cases}\bar{f}_{s, k-n_{f_{a}}+i}^{-1} & n_{f_{a}}-k \leq i \leq n_{f_{s}}+n_{f_{a}}-k \\
0 & \text { else }\end{cases}
\end{aligned}
$$

The equations (29)-(40) together constitute a complete and explicit description of the gradient $\psi(t, \theta)=$ $\frac{d}{d \theta} \hat{y}(t \mid \theta)$ which may be used in an implementation of the search algorithm (7).

\subsection{Simulation Example}

To illustrate the applicability of the proposed model structure $(24)$ to identification problems involving unstable systems we will in this section present a small simulation study.

The "true" system - to be identified - is given by

$$
y(t)=\frac{b_{0}}{1+f_{1} q^{-1}+f_{2} q^{-2}} u(t)+e(t)
$$

with $b_{0}=1, f_{1}=-1.5$, and $f_{2}=1.5$. This system is unstable with poles in $0.75 \pm 0.9682 i$.

To generate identification data we simulated this system using the feedback law

$$
u(t)=r(t)-\left(-0.95 q^{-2}\right) y(t)=r(t)+0.95 y(t-2)
$$

which places the closed-loop poles in 0.8618 and 0.6382 . In the simulation we used independent, zero mean, white noise reference and noise signals $\{r(t)\}$ and $\{e(t)\}$ with variances 1 and 0.01 , respectively. $N=200$ data samples were used.

In Table 1 we have summarized the results of the identification, the numbers shown are the estimated parameter values together with their standard deviations. For comparison we have, apart from the model structure (24), used a standard output error model model structure and an ARMAX model structure. As can be seen the standard output error model structure gives completely useless estimates while the
Table 1: Summary of identification results.

\begin{tabular}{|ccccc|}
\hline Parameter & True value & OE & Modified OE & ARMAX \\
\hline$b_{0}$ & 1 & 0.6218 & 0.9898 & 1.0028 \\
& & \pm 0.0382 & \pm 0.0084 & \pm 0.0077 \\
$f_{1}$ & -1.5 & -1.0336 & -1.5008 & -1.5026 \\
& & \pm 0.0481 & \pm 0.0052 & \pm 0.0049 \\
$f_{2}$ & 1.5 & 0.7389 & 1.5035 & 1.5052 \\
& & \pm 0.0391 & \pm 0.0079 & \pm 0.0074 \\
\hline
\end{tabular}

modified output error and the ARMAX model structures give very similar and accurate results.

From Table 1 it is also clear that for the ARMAX case the bias (due to the feedback) is negligible in this example, even though the noise model is incorrect. The reason for this is the high SNR. We conclude that direct closed-loop identification does not automatically lead to useless identification results due to feedback-induced bias errors.

\section{An Alternative Box-Jenkins Model Structure}

The trick to include a "fake" noise model in the output error model structure is of course also applicable to the Box-Jenkins model structure. The alternative form will in this case be

$$
y(t)=\frac{B(q)}{F(q)} u(t)+\frac{F_{a}^{*}(q) C(q)}{F_{a}(q) D(q)} e(t)
$$

with the corresponding predictor

$$
\begin{aligned}
\hat{y}(t \mid \theta) & =\frac{F_{a}(q) D(q) B(q)}{F_{a}^{*}(q) C(q) F(q)} u(t)+\left(1-\frac{F_{a}(q) D(q)}{F_{a}^{*}(q) C(q)}\right) y(t) \\
& =\frac{D(q) B(q)}{C(q) F_{a}^{*}(q) F_{s}(q)} u(t)+\left(1-\frac{D(q) F_{a}(q)}{C(q) F_{a}^{*}(q)}\right) y(t)
\end{aligned}
$$

An explicit expression for the gradient filters for this predictor can be derived quite similarly as in the output error case, albeit that the formulas will be even messier. For the sake of readability we skip the details. 


\section{Conclusions}

In this paper we have proposed new versions of the well known output error and Box-Jenkins model structures that can be used also for identification of unstable systems. The new model structures are equivalent to the standard ones, as far as number of parameters and asymptotical results are concerned, but guarantee stability of the predictors.

\section{References}

[1] U. Forssell. Properties and Usage of Closed-loop Identification Methods. Licentiate thesis LIUTEK-LIC-1997:42, Department of Electrical Engineering, Linköping University, Linköping, Sweden, September 1997.

[2] M. Gevers. Towards a Joint Design of Identification and Control. In H. L. Trentelman and J. C. Willems, editors, Essays on Control: Perspectives in the Theory and its Applications, pages 111-151. Birkhäuser, 1993.

[3] F. R. Hansen, G. F. Franklin, and R. Kosut. Closed-loop identification via the fractional representation: experiment design. In Proceedings of the American Control Conference, pages 1422-1427, Pittsburg, PA, 1989.

[4] L. Ljung. System Identification: Theory for the User. Prentice-Hall, 1987.

[5] L. Ljung. Information contents in identification data from closed loop operation. In Proc. 32nd IEEE Conference on Decision and Control, pages 2242-2247, San Antonio, TX, December 1993.

[6] L. Ljung. Identification in closed loop: some aspects on direct and indirect approaches. In Preprints of the 11th IFAC Symposium on System Identification, volume 1, pages 141-146, Fukuoka, Japan, July 1997.

[7] T. Söderström and P. Stoica. System Identification. Prentice-Hall International, 1989.
[8] P. M. J. Van den Hof and R. J. P. Schrama. An Indirect Method for Transfer Function Estimation from Closed Loop Data. Automatica, 29(6):1523-1527, 1993.

[9] P. M. J. Van den Hof and R. J. P. Schrama. Identification and Control - Closed-loop Issues. Automatica, 31(12):1751-1770, 1995.

[10] P. M. J. Van den Hof, R. J. P. Schrama, R. A. de Callafon, and O. H. Bosgra. Identification of normalized coprime factors from closed-loop experimental data. European Journal of Control, 1(1):62-74, 1995. 